\title{
Fixation of greater tuberosity fracture in rare unstable bilateral glenohumeral joint dislocations with bilateral fractures of the greater tuberosities: A case report
}

\author{
RP Shah Kalawar, BP Shrestha, GP Khanal, P Chaudhary, R Rijal, R Maharjan, SR Paneru \\ Department of Orthopaedics, B.P. Koirala Institute of Health Science, Dharan, Nepal
}

\begin{abstract}
Introduction: Unstable dislocations of the bilateral glenohumeral joint with fractures of both greater tuberosities are very rare. Case presentation: A 48-year-old gentleman sustained a bilateral anterior dislocation of his glenohumeral joint with fractures of the greater tuberosities on both sides after an episode of seizure. Attitude of both shoulders were abducted and externally rotated. Radiological examination revealed the dislocations of bilateral glenohumeral joint and also the fractures of the bilateral greater tuberosities. Closed reduction and shoulder derotation immobilizer was applied. The Reduction was unstable and needed fixation of both sides greater tuberosity with cannulated cancellous screws. The shoulders were immobilized for 3 weeks and subsequent rehabilitation yielded a good outcome. Results at 6 months follow-up were satisfactory with normal range of motion and no redislocations occurring. To our knowledge, this is the first reported case of bilateral unstable glenohumeral joint dislocation associated with fractures of both greater tuberosities in a middle aged man which was unstable needing fixation of greater tuberosities fractures. Conclusion: Bilateral glenohumeral joint dislocations with bilateral fractures of the greater tuberosities may be unstable needing fixation of greater tuberosity fracture.
\end{abstract}

Keywords: Glenohumeral joint dislocation, greater tuberosity fracture

\section{Introduction}

Bilateral glenohumeral joint dislocations are rare although the glenohumeral joint is the most frequently dislocated joint ${ }^{1}$. Sports injuries, epileptic seizures, electrical shock, or electroconvulsive therapy without muscle relaxation usually leads such dislocations $s^{2-6}$. Bilateral fracture-dislocation of glenohumeral joint is even rarer, with only few cases reported. Seizures had been incriminated for bilateral anterior shoulder fracture dislocation ${ }^{7}$. We are reporting an unusual 
case of dislocations of the bilateral glenohumeral joint with fractures of the bilateral greater tuberosities following a seizure episode in a 48 year-old gentleman.

\section{Case presentation}

A 48 year-old gentleman was brought to the emergency department of BPKIHS complaining of acute pain and deformity of both shoulders after an episode of seizure. His seizure was in control. He had no history of previous shoulder dislocation. Physical examination revealed fullness over the anterior aspect and flattened contour of both shoulders below the tip of the acromion suggestive of bilateral shoulder dislocations. Both arms were abducted and externally rotated. There was no neurovascular deficit on both sides. The anteroposterior view bilateral shoulder revealed dislocations of the bilateral glenohumeral joints as well as fractures of both greater tuberosities (Figure 1). Clinicoradiologically, he was diagnosed as having dislocations of the bilateral glenohumeral joint with fractures of both greater tuberosities. Prompt closed reduction was done and shoulder derotation immobilizer was applied.

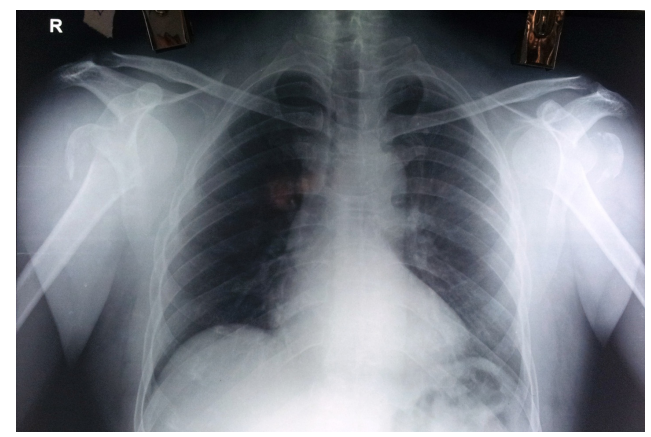

Figure 1: Pre-operative radiograph: Bilateral glenohumeral dislocation with fractures of bilateral greater tuberosities.

The joints dislocated with minor degree of movements even with well applied shoulder derotation immobilizer. So, these reductions were unstable and needed fixation of both sides greater tuberosity with cannulated cancellous (CC) Screw. Both shoulder joints were immobilized for 3 weeks and subsequent rehabilitation yielded a good outcome. At 3 months follow-up, he was very happy with stable and satisfactory range of motion of both shoulders (Figure $2 \mathrm{a}$ and $2 \mathrm{~b}$ ).

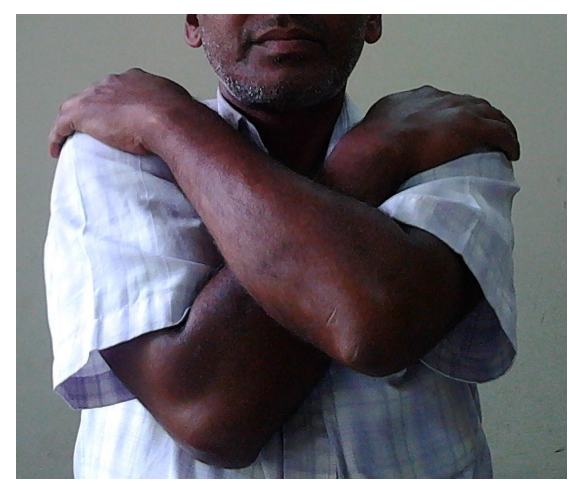


Figure $2 \mathrm{a}$ and 2b: Three months postoperative clinical photographs showing satisfactory range of motion.

Radiographs of both shoulders showed union of both greater tuberosity fracture.

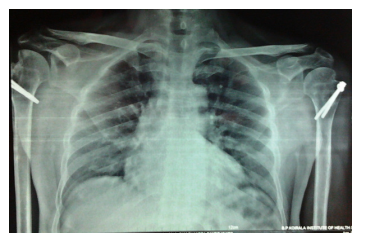

$3(a)$

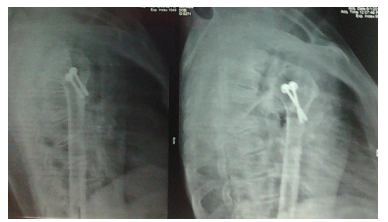

3 (b)
Figure $3 a$ and 3b: Three months postoperative anteroposterior and transthoracic lateral radiographs showing union of bilateral greater tuberosities fracture

\section{Discussion}

Anterior dislocation of glenohumeral joint is the most common major joint dislocation encountered in the emergency department which may be probably because of the position naturally adopted by the upper extremity during a fall. The injury mechanism is forced extension, abduction, and external rotation.

Our case experienced anterior dislocations of the bilateral glenohumeral joint with fractures of the greater tuberosities on both sides following an episode of seizure. Bilateral glenohumeral joint dislocations are rare and almost always posterior ${ }^{1}$. Such dislocations are usually caused by sports injuries, epileptic episodes, seizures, electrocution including electroconvulsive therapy, drug overdose, neuromuscular disorders, or severe emotional disturbances ${ }^{2,3}$. However, anterior dislocations of bilateral glenohumeral joints are very rare and only about 30 cases are reported in the literature, 15 of which are of fracture-dislocation. None of the reported fracture dislocations of bilateral glenohumeral joints were acutely unstable. Most were due to violent trauma from sports injuries and electrocution but also seizures and diabetic nocturnal hypoglycemia were incriminated ${ }^{7}$.

Bilateral anterior shoulder dislocation was first described in 1902 in a person with muscular contraction caused by overdose of camphor $^{2}$. Bilateral fracture-dislocation is even rarer, with only few reports in the literature. Associated fracture of the greater tuberosity occurs in $15 \%$ of the anterior dislocation cases and it signifies an associated rotator cuff tear ${ }^{10}$. Age over forty years, the first episode of dislocation and the mechanism of injury are three significant clinical factors associated with occurrence of fractures in anterior dislocations of bilateral glenohumeral joint ${ }^{10}$. Dinopoulos et al reported a 76-year-old woman who experienced an anterior dislocation of both shoulders with a 3-part fracture of the right proximal humerus following a fall on outstretched $\operatorname{arms}^{8}$. The outcome was satisfactory after closed reduction and 
shoulder immobilization. Our case of post seizure anterior dislocations of bilateral glenohumeral joint with bilateral fractures of the greater tuberosity is rare. Fracture of the greater tuberosity occurs in approximately $10 \%$ of dislocations and is usually associated with the humeral head in a subglenoid, low subcoracoid, or subclavicular position. In these cases, reduction maneuver may depend on the position of the humeral head. The greater tuberosity is displaced in the approximately $15 \%$ of all anterior dislocation of shoulder ${ }^{10}$. Rotator cuff tear is almost sure when the fracture of the greater tuberosity is displaced. Functional impairment usually occurs if the greater tuberosity is not reduced anatomically. Our patient underwent closed reduction but as it was unstable we had to do open reduction and internal fixation with $\mathrm{CC}$ Screw for greater tuberosity fracture. He was able to resume his daily activities after 2 months. Although most of the anterior dislocations of shoulder are easily reduced by classical closed maneuvers under adequate sedation and analgesia, the interposition of biceps tendon, subscapularis or a greater tuberosity fragment can prevent reduction. General anaesthesia is indicated for these cases. Reported complications of difficult reductions of shoulder dislocations are fractures of proximal humerus, brachial plexus injury, soft-tissue injuries, vascular injury, and recurrent dislocations ${ }^{9}$.
Bilateral glenohumeral joint dislocations with bilateral fractures of the greater tuberosities may be unstable needing fixation of greater tuberosity fracture.

\section{Conclusion}

Anterior dislocation of the bilateral glenohumeral joints with associated bilateral fractures of the greater tuberosities is an extremely rare injury. Clinical examination help to diagnose the dislocation but radiographs are mandatory to make an accurate and early diagnosis. Non-displaced and minimally displaced fractures of greater tuberosity are typically successfully treated conservatively followed by shoulder immobilization. Surgery is recommended for unreduced dislocation, displaced fracture or unstable reduction. Subsequent physiotherapy usually restores satisfactory outcome after both surgical and nonsurgical treatment.

\section{References}

1. Brown RJ. Bilateral dislocation of the shoulders. Injury. 1984; 15: 267e273.

2. Dunlop CC. Bilateral anterior shoulder dislocation: a case report and review of the literature. Acta Orthop Belg. 2002; 68: $168 \mathrm{e} 170$.

3. Mehta MP, Kottamasu SR. Anterior dislocation of the shoulders with bilateral 
brachial plexus injury. Ann Emerg Med. 1989; 18: 589e591.

4. Becker R, Weyand F. Die seltene, doppelseitige hintere Schulterluxation. Unfallschirurg. 1990; 93: 66e68.

5. Blasier RB, Burkus JK. Management of posterior fracture dislocations of the shoulder. Clin Orthop Relat Res. 1988; 232: 197e204.

6. Martens C, Hessels G. Bilateral posterior four-part fracture dislocation of the shoulder. Acta Orthop Belg. 1995; 61(3): $249 \mathrm{e} 254$.
7. Devalia KL, Peter VK. Bilateral post traumatic anterior shoulder dislocation. J Postgrad Med. 2005; 51(1): $72 \mathrm{e} 73$.

8. Dinopoulos HT, Giannoudis PV, Smith $\mathrm{RM}$, et al. Bilateral anterior shoulder fracture-dislocation. A case report and a review of the literature. Int Orthop. 1999; 23(2): $128 \mathrm{e} 130$.

9. Canale ST, ed. Campbell's Operative Orthopaedics. 10th ed. St. Louis, MO: Mosby; 2003.

10. Ferkel RD, Hedley AK, Eckardt JJ. Anterior fracture dislocations of the shoulder: pitfalls in treatments. $J$ Trauma. 1984; 24: $363 e 367$. 